\title{
Study of dimuon rare beauty decays with ATLAS and CMS
}

\author{
A. Policicchio ${ }^{\mathrm{a}}$, G. Crosetti \\ Università della Calabria and INFN Cosenza, Ponte P. Bucci Cubo 31c, 87036 Arcavacata di Rende - Cosenza, Italy \\ representing the ATLAS and CMS Collaborations
}

Received: 7 January 2008 /

Published online: 19 April 2008 - (C) Springer-Verlag / Società Italiana di Fisica 2008

\begin{abstract}
The LHC experiments will perform sensitive tests of physics beyond the standard model (BSM). The investigation of decays of beauty hadrons represents an alternative approach in addition to direct BSM searches. The ATLAS and CMS efforts concentrate on those $B$-decays that can be efficiently selected already at the first and second level trigger. The most favorable trigger signature will be for $B$-hadron decays with muons in the final state. Using this trigger, ATLAS and CMS will be able to accommodate very high statistics in the rare decay sector. These are purely dimuon decays, and families of semimuonic exclusive channels. Already with data corresponding to an integrated luminosity of $1 \mathrm{fb}^{-1}$, the sensitivity in the dimuon channels will be comparable to present measurements (world average). The strategy is to carry on the dimuon channel program up to nominal LHC luminosity. In particular the $B_{s} \rightarrow \mu \mu$ signal with $\sim 5$ sigma significance can be measured combining low luminosity $10^{33} \mathrm{~cm}^{-2} \mathrm{~s}^{-1}$ samples with those of one year of LHC operation at a luminosity of $10^{34} \mathrm{~cm}^{-2} \mathrm{~s}^{-1}$.
\end{abstract}

PACS. 13.30.Ce; 13.20.He

\section{Introduction}

Rare leptonic and semileptonic $B$-decays, produced by FCNC transitions, are forbidden at the tree level in the standard model (SM). These decays occur at the lowest order only through one-loop "penguin" and "box" diagrams. The branching ratios of these decays are very small: from $4 \times 10^{-5}$ for the rare radiative decay $B_{d}^{0} \rightarrow K^{*} \gamma$ to $10^{-15}$ for the rare Cabibbo suppressed leptonic decay $B_{d}^{0} \rightarrow e^{+} e^{-}$.

The careful investigation of rare $B$-decays is mandatory for testing ground of the standard model and offers a complementary strategy in the search of new physics. The probing of loop-induced couplings provide a means of testing the detailed structure of the SM at the level of radiative corrections. In particular, FCNC involving $b \rightarrow s, d$ transitions and $B \rightarrow l l$ decays, provide an excellent probe of new indirect effects by yielding informations on the masses and coupling of the virtual particles running in the loops. In SUSY models, the branching fraction for $B_{s(d)}^{0} \rightarrow \mu^{+} \mu^{-}$has a strong dependence on $\tan \beta$. A precise measurement of such decays will allow to constrain the supersymmetric extensions of the SM.

To date the decay modes $B_{s(d)}^{0} \rightarrow \mu^{+} \mu^{-}$have not yet been observed. The current best upper limits on the branching ratio come from the D0 [1] and CDF [2] collaborations and are $9.3 \times 10^{-8}$ and $5.8 \times 10^{-8}$ respec-

\footnotetext{
a e-mail: antonio.policicchio@cern.ch
}

tively at $95 \%$ C.L. The searches for rare $B$ decays at the $B$-factories CLEO, Belle and BaBar have no sensitivity to $B_{s}$ decays.

In the last years the $B$-factories BaBar and Belle presented the first results for $B \rightarrow\left(K^{*}, K\right) l^{+} l^{-}$branching ratios and forward-backward asymmetry $\left(A_{\mathrm{FB}}\right)$ in these rare semileptonic decays $[3-5]$ but those are still affected by large statistical errors.

In this report we pay attention to the (semi)leptonic decays with $\mu^{+} \mu^{-}$pairs in final states where ATLAS and CMS can give a significant contribution. We discuss the simulation results and the perspectives of measurements. The SM branching ratios of the decays studied can be found in Table 1. The perspective of measurement of rare beauty decays have been presented during the SUSY07 conference also by the LHCb collaboration [6].

Table 1. Standard model branching ratios for rare $B$-decays into a $\mu^{+} \mu^{-}$final state

\begin{tabular}{lcc}
\hline Decay channel & Branching ratio & Ref. \\
\hline$B_{s}^{0} \rightarrow \mu^{+} \mu^{-}$ & $3.4 \times 10^{-9}$ & {$[7]$} \\
$B^{+} \rightarrow K^{+} \mu^{+} \mu^{-}$ & $3.5 \times 10^{-7}$ & {$[8]$} \\
$B^{+} \rightarrow K^{*+} \mu^{+} \mu^{-}$ & $\sim 10^{-6}$ & {$[8]$} \\
$\Lambda_{b} \rightarrow \Lambda \mu^{+} \mu^{-}$ & $2.0 \times 10^{-6}$ & {$[9,10]$} \\
$B_{d}^{0} \rightarrow K^{0 *} \mu^{+} \mu^{-}$ & $1.3 \times 10^{-6}$ & {$[11,12]$} \\
$B_{s}^{0} \rightarrow \phi \mu^{+} \mu^{-}$ & $\sim 10^{-6}$ & {$[11,12]$} \\
\hline
\end{tabular}




\section{Trigger strategies for rare decays}

Details of the ATLAS and CMS experiments can be found in $[13,14]$.

\subsection{The ATLAS trigger}

ATLAS has a three level trigger system [15] which reduces the $40 \mathrm{MHz}$ bunch crossing rate to about $100 \mathrm{~Hz}$ of events to be recorded. The first level trigger (LVL1) is hardware-based and makes a fast decision (in $2.5 \mu \mathrm{s}$ ) about which events are interesting for further processing. Coarse granularity informations from calorimeter and muon spectrometer are used to identify region of interest (RoI) of the detector which contain interesting signals (high energy electrons, muons and taus and jets with large transverse or missing energy). The RoIs are used to guide the later stages of the trigger. After LVL1 the trigger rate will be reduced to less than $75 \mathrm{kHz}$.

The high level trigger (HLT) is software-based and is split into two levels. At the level 2 (LVL2) the full granularity of the detector is used to confirm the LVL1 decisions and then to combine informations from different sub-detectors within the LVL1 RoIs. Fast algorithms are used for the reconstruction at this stage and the rate is reduced to $\sim 2 \mathrm{kHz}$ with an average time of execution of $\sim 10 \mathrm{~ms}$. At the level 3, the event filter (EF), the whole event is available and offline-like algorithms are used with better alignment and calibration informations to form the final decision. The rate is reduced to $100 \mathrm{~Hz}$ with an execution time of $\sim 1 \mathrm{~s}$.

\subsubsection{ATLAS trigger for rare decays}

The $B$-trigger is expected to account for $5 \%-10 \%$ of the total trigger resources. The core of the $B$-trigger is the LVL1 muon trigger which is based on the measurement of the muon transverse momentum $\left(p_{\mathrm{T}}\right)$. The efficiency of the muon trigger is expected at about $90 \%$ above $p_{\mathrm{T}}>7 \mathrm{GeV}$. The dimuon LVL1 trigger (two muons with $p_{\mathrm{T}}>6 \mathrm{GeV} / c$ ), used for rare decay selection, is expected to have a rate of about $500 \mathrm{~Hz}$ at $10^{33} \mathrm{~cm}^{-2} \mathrm{~s}^{-1}$.

The LVL1 dimuons will be confirmed at the LVL2 firstly in the muon system by means of the precision tracking chamber and then by combining muon and inner detector tracks. Finally the two muons can be combined and mass cuts are applied. At the EF the tracks are refitted in the RoIs and vertex reconstruction is performed. Cuts are applied on decay length and invariant $B$-hadron mass. For $B_{s}^{0} \rightarrow \mu^{+} \mu^{-}$events containing two muons with $p_{\mathrm{T}}>6 \mathrm{GeV} / c$, the expected trigger efficiency is $46 \%$.

\subsection{The CMS trigger}

CMS has a two level trigger [14] which reduces the bunch crossing rate down to about $150 \mathrm{~Hz}$ for recording. The level-1 (L1) trigger uses muon detector and calorimeter informations and is hardware-based with an output rate of about $100 \mathrm{kHz}$ and a latency of $3.5 \mu \mathrm{s}$. The HLT is software-based with the required $150 \mathrm{~Hz}$ output rate. It uses reconstruction algorithms similar to the offline with a mean execution time per event of about $40 \mathrm{~ms}$. To speed up reconstruction in the HLT, a partial track reconstruction is performed: the difference in track resolution becomes negligible after 5-6 hits are used in the track fit.

\subsubsection{CMS trigger for rare decays}

As in ATLAS, the CMS trigger for $B$ events uses single and dimuon triggers. The L1 dimuon trigger has a low $p_{\mathrm{T}}$ threshold of $3 \mathrm{GeV} / c$ which ensures a high efficiency for events with two muons in the final state with a rate of $0.9 \mathrm{kHz}$ at $2 \times 10^{33} \mathrm{~cm}^{-2} \mathrm{~s}^{-1}$. At the HLT the L1 decision will be confirmed using the full muon system and an improved momentum measurement with the tracker. Primary vertex (PV) reconstruction is available from the pixel detector and also track reconstruction is performed in cones around the L1 muons using the partial track reconstruction. The exclusive rare decay is then reconstructed and cuts on the invariant mass, vertex fit quality and decay length are applied. The expected global trigger efficiency for $B_{s}^{0} \rightarrow \mu^{+} \mu^{-}$selection is about $41 \%$.

\section{Muonic decays in ATLAS and CMS}

As purely leptonic $B$-decays are theoretically very clean, they provide an ideal channel for seeking indirect hints of new physics effects. However, they are very difficult to observe because of their small branching ratio (see Table 1). Assuming the standard model branching ratio, most probably they will not be observed by other experiments before the LHC data taking. ATLAS and CMS will start sensitive measurements at $10^{33} \mathrm{~cm}^{-2} \mathrm{~s}^{-1}$. Even at high design luminosity $\left(10^{34} \mathrm{~cm}^{-2} \mathrm{~s}^{-1}\right)$ the trigger for $B \rightarrow \mu^{+} \mu^{-}$decay is not problematic.

A good background rejection is necessary for the signal selection. The main contributions to the background [16] come from the processes $b \bar{b}(b \bar{b} b \bar{b}, b \bar{b} c \bar{c}) \rightarrow X \mu^{+} \mu^{-}$with the muons originating mainly from semi-leptonic $b$ and $c$ quark decays. This background can be estimated by extrapolating the Tevatron data on heavy quark production to the LHC energies. In addition to the combinatorial background, two and three body decays of $B$ meson where two of the final state particles are $K^{ \pm}, \pi^{ \pm}$or $\mu^{ \pm}$, may leak into the signal region. Although the rate for misidentification of kaons or pions as muons, due to punchthrough or decay in flight, is low (in ATLAS is of the order of $0.5 \%$ ), the investigation of such decays has been done, because of the small branching ratio of the $B \rightarrow \mu^{+} \mu^{-}$decay.

The event selection relies on topological variables related to the PV, the muon candidates and the $B_{s}$ secondary vertex and is very similar in both experiments $[17,18]$. Simple cuts can be applied to distinguish the combinatorial background from the signal:

- $B$-hadron invariant mass;

- secondary vertex length and quality; 
- pointing of $B$-hadron momentum to PV;

- track isolation.

Table 2 summarizes the mass resolution and the proper time resolution obtained on the signal Monte Carlo event sample for CMS and ATLAS.

The expected number of signal and background events after $30 \mathrm{fb}^{-1}$ are summarized in Table 3 . This will allow to set a stringent constraint on new physics models.

Figure 1 shows the ATLAS expectation for the measurement of the $B_{s}^{0} \rightarrow \mu^{+} \mu^{-}$branching ratio as a function of the integrated luminosity (or equivalently as a function of time). The SM expectation can be reached with $\mathrm{a} \sim 5$

Table 2. Mass and proper time resolution obtained on the $B_{s}^{0} \rightarrow \mu^{+} \mu^{-}$signal Monte Carlo event sample for CMS and ATLAS

\begin{tabular}{lcc}
\hline Experiment & Mass res. $\left(\mathrm{GeV} / c^{2}\right)$ & Proper time res. (fs) \\
\hline ATLAS & 0.084 & 91 \\
CMS & 0.036 & 95 \\
\hline
\end{tabular}

Table 3. Expected number of $B_{s}^{0} \rightarrow \mu^{+} \mu^{-}$signal and background events after $30 \mathrm{fb}^{-1}$

\begin{tabular}{lcc}
\hline Experiment & Signal events & BG events \\
\hline ATLAS & 21.0 & $60 \pm 36$ \\
CMS & 18.3 & $42_{-42}^{+66}$ \\
\hline
\end{tabular}

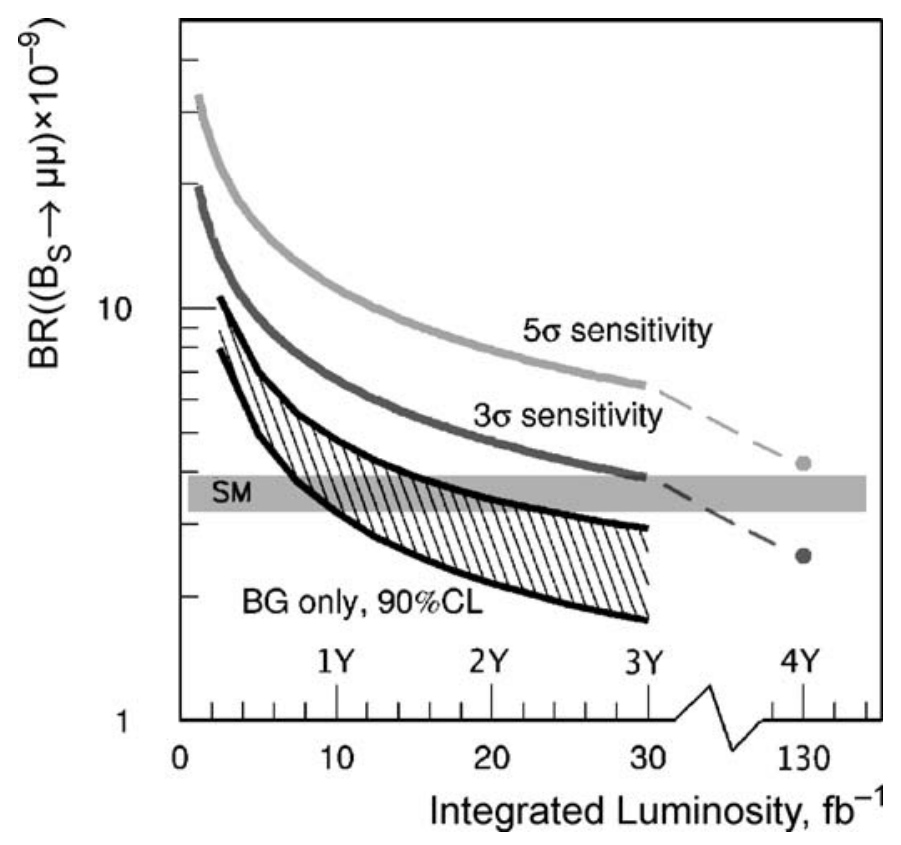

Fig. 1. ATLAS perspective for the measurement of the $B_{s}^{0} \rightarrow$ $\mu^{+} \mu^{-}$branching ratio as a function of the integrated luminosity (or equivalently as a function of the time). The shaded band shows the uncertainty in the BG level estimation sigma significance combining low luminosity $10^{33} \mathrm{~cm}^{-2} \mathrm{~s}^{-1}$ samples with those of one year of LHC operation at the nominal luminosity of $10^{34} \mathrm{~cm}^{-2} \mathrm{~s}^{-1}$.

\section{Semimuonic decays in ATLAS}

Thanks to the dimuon final state, the semimuonic decays, as the purely dimuonic ones, are easy to select at the trigger level. The observation of semileptonic decays gives access to a number of observables. The precise measurements of such observables could give very interesting informations for new physics reach. $A_{\mathrm{FB}}$ is one of the most promising parameters.

The small branching ratios of semimuonic decays require a powerful background rejection. Semileptonic decays with $c \bar{c}$ resonances decaying into two muons represent an irreducible background source. A cut on the dimuon invariant mass around the nominal values for resonances removes this background. Combinatorial background arises from muons originating mainly from semileptonic decays of $b$ and $c$ quarks. The non-resonant background $B \rightarrow K \pi \mu \mu$, which is an irreducible background for $B \rightarrow K * \mu \mu$ decays, has not been taken into account in the present study. The cut on the invariant mass should remove a large fraction of this background. Specific decay channels can represent background sources due mainly to hadron misidentification as muons, but their contribution is expected to be less important than the previous two sources. Further studies and background productions are already ongoing and results will be updated, taking into account the missing background sources.

The event selection $[19,20]$ is related to topological variables:

- vertex quality and invariant mass of the dimuon system;

- displacement and quality of vertices and mass of the secondary hadrons;

- pointing of $B$ hadron momentum to PV.

The number of events expected after three years of data taking at low luminosity $\left(30 \mathrm{fb}^{-1}\right)$ are summarized in Table 4 . The background level estimation is only limited by the low Monte Carlo statistics available at the moment. It should be pointed out that, thanks to the muon pair in the final state, semimuonic decays will be also studied at high

Table 4. Expected number of events from semimuonic decays events and expected background events in ATLAS after $30 \mathrm{fb}^{-1}$

\begin{tabular}{lcc}
\hline Decay & Signal events & Background events \\
\hline$B^{+} \rightarrow K^{+} \mu^{+} \mu^{-}$ & 4000 & $<10000$ \\
$B^{+} \rightarrow K^{*+} \mu^{+} \mu^{-}$ & 2300 & $<10000$ \\
$\Lambda_{b} \rightarrow \Lambda \mu^{+} \mu^{-}$ & 800 & $<4000$ \\
$B_{d}^{0} \rightarrow K^{0 *} \mu^{+} \mu^{-}$ & 2500 & $<10000$ \\
$B_{s}^{0} \rightarrow \phi \mu^{+} \mu^{-}$ & 900 & $<10000$ \\
\hline
\end{tabular}




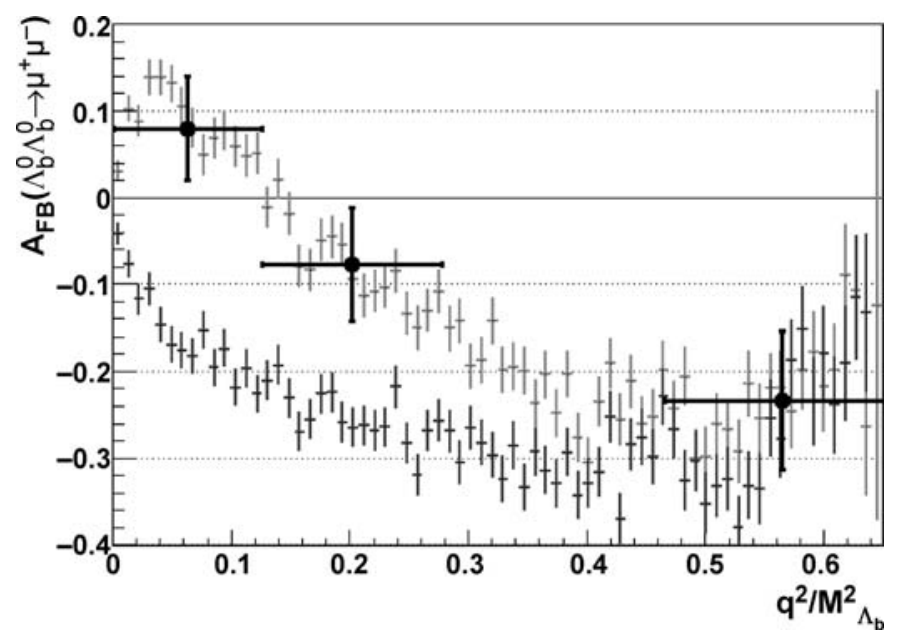

Fig. 2. Forward-backward asymmetry for $\Lambda_{b} \rightarrow \Lambda \mu^{+} \mu^{-}$as a function of the dimuon invariant mass after $30 \mathrm{fb}^{-1}$ (see Sect. 4)

luminosity, so that a larger statistics than those of Table 4 will be collected.

Experimentally, the forward-backward asymmetry has been defined as

$$
A_{\mathrm{FB}}=\frac{N_{\mathrm{F}\left[s_{1}, s_{2}\right]}-N_{\mathrm{B}\left[s_{1}, s_{2}\right]}}{N_{\mathrm{F}\left[s_{1}, s_{2}\right]}+N_{\mathrm{B}\left[s_{1}, s_{2}\right]}},
$$

where $N_{\mathrm{F}\left[s_{1}, s_{2}\right]}$ and $N_{\mathrm{B}\left[s_{1}, s_{2}\right]}$ are the numbers of positive leptons moving in the forward and backward directions of the $B$ meson in the dimuon rest frame, respectively, in the the range of the squared dimuon mass $\left[s_{1}, s_{2}\right]$. The expected precision on $A_{\mathrm{FB}}$ after $30 \mathrm{fb}^{-1}$ is presented on Fig. 2 for $\Lambda_{b} \rightarrow \Lambda \mu^{+} \mu^{-}$decay. The three dots with error bars correspond to simulated data after offline analysis. The upper point set corresponds to the theoretical SM prediction, and the lower set corresponds to a prediction within the MSSM [9]. The statistical error in the low dimuon invariant mass region, where the sensitivity of $\mathrm{AFB}$ on the form factor calculation is negligible, is at level of $6 \%$.

\section{Conclusions}

The results obtained for $B_{s}^{0} \rightarrow \mu^{+} \mu^{-}$by ATLAS and CMS are comparable and promise an interesting startup analysis with the possibility of setting tight constraints on new physics models beyond the SM. The simulation studies show that the ATLAS detector will be capable to extract signals of semimuonic $B$-decays and reach a good sensitivity to new physics beyond the SM.

Acknowledgements. We would like to thank the ATLAS and CMS Collaborations, and especially Maria Smizanska, Sergey Sivoklokov, Pavel Reznicek from ATLAS and Christina Eggel and Urs Langenegger from CMS for their useful suggestions in the preparation of this work.

\section{References}

1. D0 Collaboration, D0 Note 5344-CONF (2007)

2. CDF Collaboration, CDF Public Note 8956 (2007)

3. BaBar Collaboration, B. Aubert et al., Phys. Rev. D 73, 092001 (2006)

4. Belle Collaboration, A. Ishikawa et al., Phys. Rev. Lett. 96, 251801 (2006)

5. Belle Collaboration, K. Abe et al., hep-ex/0410006

6. LHCb Collaboration, M. Lenzi, Rare $B$ decays at LHCb, presented at SUSY07 Conf.

7. A.J. Buras, Phys. Lett. B 566, 115 (2003)

8. A. Ali et al., Phys. Rev. D 66, 034002 (2002)

9. C.H. Chen et al., Phys. Rev. D 64, 074001 (2001)

10. T.M. Aliev et al., Nucl. Phys. B 649, 168 (2003)

11. D. Melikhov et al., Phys. Rev. D 57, 6814 (1998)

12. D. Melikhov et al., Phys. Rev. D 62, 014006 (2000)

13. ATLAS Collaboration, CERN-LHCC-1999-14/15 (1999)

14. CMS Collaboration, CERN-LHCC-2006-001 (2006)

15. ATLAS Collaboration, CERN-LHCC-2003-22 (2003)

16. N. Nikitin et al., ATL-PHYS-PUB-2007-009 (2007)

17. P. Reznicek, Nucl. Phys. B Proc. Suppl. 167, 244 (2007)

18. C. Eggel et al., CMS-AN-2006-097 (2006)

19. A. Policicchio, G. Crosetti, Acta Phys. Pol. B 38, 947 (2007)

20. A. Policicchio, G. Crosetti, ATL-PHYS-PUB-2007-017 (2007) 